\title{
Routing in WSNs Powered by a Hybrid Energy Storage System through a CEAR Protocol Based on Cost Welfare and Route Score Metric
}

\author{
R. Senthilkumar, G.M. Tamilselvan, S. Kanithan, N. Arun Vignesh
}

\author{
R. Senthilkumar* \\ Department of Electrical and Electronics Engineering, \\ Roever Enginering College, Perambalur, \\ Tamil Nadu- 621212 \\ *Corresponding author: rajsen1985@gmail.com

\section{G.M. Tamilselvan} \\ Electronics and Communication Engineering, \\ Bannari Amman Institute of Technology, Sathyamangalam, \\ Tamil Nadu- 638401 \\ tamiltamil@rediffmail.com

\section{S. Kanithan} \\ Department of Electronics and Communication Engineering, \\ AVS College of Technology-Salem, \\ Tamilnadu \\ kani.rex@gmail.com

\section{N. Arun Vignesh} \\ Department of Electronics and Communication Engineering, \\ GRIET, Hyderabad \\ arunvignesh44@gmail.com
}

\begin{abstract}
Implementing a low cost, power efficient and high performance routing protocol in wireless sensor networks (WSNs) is an important requirement for transmitting a packet through network. In this paper we propose, a new cost and energy aware routing protocol (CEAR) that works based on the two metrics such as cost welfare metric and route score metric.A hybrid electrical energy storage (HEES) framework which holds numerous banks of heterogeneous electrical energy storage (EES) components to be specific battery and a ultra-capacitor is used for providing energy to the network exhibit in the WSN for routing. The simulation results shows that our proposed routing protocol routes the packet efficiently by choosing the best path that also reduces the cost and routes the packet with reduced power consumption. The quantitative metrics in terms of packet delivery ratio of 0.93 , average end to end delay of 110 secs, packet loss ratio of 0.75 , average throughput attained of $250 \mathrm{bits} / \mathrm{sec}$ and efficiency of 98-99.9\% overpowers the performance of our proposed work.
\end{abstract}

Keywords: Hybrid energy storage system; CEAR protocol, routing; cost welfare metric, route score metric.

\section{Introduction}

In a wide spectrum of applications such as wireless sensor networks (WSNs), the energy harvesting technology from the solar panels is a primary issue, which is to be tackled for grant effectiveness. The harvested energy should be stored and controlled using appropriate devices to power the sensor nodes present in the wireless networks [4]. Batteries are currently used for powering in most wireless sensors, where the power requirements are modest. The primary 
batteries are usually chosen for their high energy densities, low leakage rates and low cost. For sensor node applications, lifetimes of battery is at least a year is desirable [8].A hybrid energy framework, shaped by consolidating various energy storage systems (e.g., battery, ultra-capacitor, flywheel, power module, and so on.) and generators (e.g., smaller scale turbine, wind turbine, PV board, and so on.), has turned out to be a solution to meet the energy/power prerequisites with enhanced adaptability, unwavering quality and cost proficiency [5].Subsequent to the framework arrangement and conduct of the hybrid framework are turning out to be more unpredictable, the streamlined administration and control of a hybrid energy framework is still a testing errand [20]. To accomplish the ideal solution for this energy administration issue, research activity in recent years has focused on the design and development of novel hybrid energy storage systems that use renewable energy technologies [3].

Hybrid energy storage systems (HESS) are essential, when single renewable energy technologies need to be utilized in a more effective way and if they need to be more appealing from a financial point of view [10]. The significant point of interest of this hybrid energy storage systems is that they can, at the same time guarantee distinctive types of energy, for instance electricity, hot water, heating/cooling capacity, and so on, which is by and large an interest in diverse sorts of building offices [25]. Another ecological aspect of this HESS is they are critical and absolutely contemplated with respect to present atmosphere issues and the ones that anticipate us. Regardless of the way that the structure of HESSs is reasonably fundamental, those systems have been generally connected [6].

The fundamental idea in HESS is to utilize ultra-capacitors (UCs) as a right hand energy storage system along with the batteries to enhance the execution of the whole energy storage systems, in terms of effectiveness, unwavering quality, and element reaction [29]. UCs give quick and effective energy conveyance and long cycle-existence without any concoction response included. Likewise, the state of charge (SOC) of a UC can be precisely acquired in light of the fact that its $\mathrm{SOC}$ is relative to the square of the cell voltage [7]. The scope of $\mathrm{UC}$ working temperature $\left(-40\right.$ to $\left.+70^{\circ} \mathrm{C}\right)$ is too more extensive than that of batteries. The essential disservice of UCs is their moderately low energy thickness contrasted with batteries [9], [17]. Hence hybridization of batteries (and/or power modules) and UCs is considered in nature to be the best utilization of UCs without a doubt applications [2]. Such stored energies are efficiently managed and supplied to the wireless sensor nodes when there is a demand of power [13]- [14].

In order to operate the self-sustainable WSNs in isolated places, the energy aware routing protocol forwards the data packets through network, while extending the lifetime of network [11]. Before its capacity falls below than $80 \%$ of its initial rate capacity, the nodes are still limited by the number of charge/discharge cycles of a Rechargeable Battery (RB) [16], [25]. Wireless communications require more energy consumption than sensing and computing tasks [28]. The HESS is composed by RB and a super-capacitor (SC). A power management device is utilized to control all the operations in the energy system [23].

To reduce the power consumption in the sensor nodes and manage the power between the sensor nodes, this paper we propose a Cost and Energy Aware Routing (CEAR) protocol, which reduces the cost and power consumption by the evaluation of two metrics namely cost welfare metric and route source metric. The performance evaluation are conducted based on packet delivery ratio, average end to end delay, packet loss, packet loss ratio, average throughput. The remaining of this paper is organized as follows: Section 2 presents some the recent works related to our proposed work. Section 3 presents the detailed explanation about our proposed methodology. Section 4 presents the results obtained by our proposed routing protocol followed by the conclusion in section 5 . 


\section{Related work}

Some of the most recent works related to our proposed routing method is explained as follows:-

Zhang, Bo, et al. [27] described a broadly useful multi-hop WSN architecture capable of supporting time-critical CPS systems utilizing energy harvesting. At that point they exhibited a set of Harvesting Aware Speed Selection (HASS) algorithms. That boosts the base energy save for every one of the nodes in the network, accordingly guaranteeing highly strong performance under emergency or fault driven circumstances. At that point they introduced an ideal centralized solution, alongside a distributed solution and actualized a CPS-specific trial procedure.

Arabinda Nandha et.al [19] explained a work distance, energy and the angle formed by the node with the base station are taken as input parameters. The Mamdani fuzzy inference system is used to select the chance of a sensor node to become a cluster head $(\mathrm{CH})$. A-Star search algorithm is used to locate an optimum route from source to sink node. The data packets are routed on the selected path. The A-Star search algorithm finds the shortest route path from source node to sink node. This approach is admissible, complete, and optimal one as it uses A-Star algorithm.

Selvi et.al [24] described a technique called the rule based clustering for routing model provides better performance in terms of network lifetime than the other existing techniques since they consume more energy during the formation of clusters and finding the shortest path. Moreover, additional overhead on the cluster head selection is tackled also using rules in this proposed model in an efficient manner by building balanced clusters. The main advantage of the proposed approach is that it extends the lifetime of the network and increases the throughput, energy efficiency, link quality, and scalability.

Roshani H.Padyal et.al [21] implemented an Opportunistic based neighbor coverage routing. In this hop-by-hop opportunistic routing is done by selecting a forwarding node on the base of the current load on the node, energy, and neighbor coverage information. In this choosing of the reliable node in the path, an establishment is based on load information table. Proposed protocols minimize energy consumption and enhance network performance as compared to existing routing protocol.

Suneet Kumar Gupta et.al [22] discussed an energy efficient and balanced route generation algorithm is proposed with considering both energy efficacy and energy balancing issues. Here, we consider the distance and residual energy of the nodes as energy efficiency parameters and energy is balanced by diverting the incoming traffic to other nodes having comparably lower incoming traffic. To develop the routing schedule, we have applied Genetic Algorithm which can quickly compute the routing schedule as per the current state of the network. It is observed that the performance of proposed algorithm is better than existing algorithm in terms of first node die and energy consumption in the network.

Nilanjan Mukherjee and Dani Strickland [18] actualized a particular boost multilevel buck converter based topology that coordinate the half breed second life batteries with the grid tie inverter. A reasonable module based distributed control architecture was introduced to totalize each converter modules according to its characteristics autonomously. The converter and control architecture were observed to be adaptable to coordinate different batteries with an inverter dc link. Modeling, investigation and test approval were performed on a solitary stage secluded half breed battery energy storage system model to comprehend the operations of the control technique with different cross breed battery configurations.

Abeywardana, DB Wickramasinghe,et al. [1] built up a battery-supercapacitor based cross breed energy storage system (HESS) comprising of two DC/AC support converters, battery, supercapacitors, matrix connection, state of charge (SOC) estimation and an associated control 
systems were tentatively verified and further moved forward. The switching frequency current swell component in both battery and supercapacitor currents were reduced by stage shifted interleaved operation. In the meantime, the control system keeps up the supercapacitor voltage in pre-characterized esteem and the battery's SOC. The estimation can be finished by a broadened Kalman channel kept up within the specified SOC limits.

Qing Xie et al. [26] formally described the worldwide charge allocation issues in HEES systems, in particular, conveying a specified level of incoming energy to a subset of destination EES (Electrical Energy Storage) banks, with the goal that efficiency of most extreme charge allocation was achieved. To set the worldwide charge allocation efficiency, the issue was figured as a mixed integer nonlinear program with the objective function, where the constraints capturing key requirements and features of the system such as the energy conservation law, power conversion losses in the chargers, rate capacity and self-discharge effects in the EES components.

Huang, et al. [12] actualized an energy sharing controller in view of distributed battery energy storage system architecture. The re-designed DC-DC control stage and the energy sharing controller are used to achieve SOC balancing among the battery cells while in the meantime giving DC bus voltage direction to whatever is left of the system or load. As a result, there is no requirement for two autonomous converter systems for cell SOC balancing and DC bus voltage control. This prompts reduced design complexity of the battery energy storage system. The proposed energy sharing controller tends to the battery cells' SOC imbalance issue from the root by changing the discharge/charge rate of each battery cell.

Zhang, Bo, et al. [27] described a broadly useful multi-hop WSN architecture capable of supporting time-critical CPS systems utilizing energy harvesting. At that point they exhibited aset of Harvesting Aware Speed Selection (HASS) algorithms. That boosts the base energy save for every one of the nodes in the network, accordingly guaranteeing highly strong performance under emergency or fault driven circumstances. At that point they introduced an ideal centralized solution, alongside a distributed solution and actualized a CPS-specific trial procedure.

\section{Routing in WSN using CEAR protocol}

Mobilizing the natural resources such as solar energy in the maximum range and storing the energy which is used to power the sensor nodes present in the wireless sensor network (WSN) is done by the design of hybrid accumulator that consist of a battery and a ultra-capacitor is implemented in the previous work. Also the stored energy between battery and the ultracapacitor is controlled and managed using an adaptive power organizing algorithm in the previous work. The power supplied to the sensor nodes by means of the battery and ultra-capacitor in the hybrid accumulator depending on the demand of power. The success of wireless sensor networks and their pervasive use is somehow constrained by energy supply which generally provided by batteries. The network lifetime depends on the balance between energy consumption and reliable delivery of data packets. The nodes in the wireless networks powered by a hybrid energy storage system (HESS) require a specific management unit to control the different energy flows. However, limited battery life has been a barrier for widespread deployment of wireless networks. To overcome such issues, in this paper we proposed a Cost and Energy Aware Routing (CEAR) protocol for efficient and inexpensive routing. The architecture of our proposed method is shown in figure 1. From figure 1 we can see that the sensor nodes present in the wireless sensor network is powered by means of renewable energy source such as solar energy extracted from the PV panels. The extracted energy is stored and supplied to the WSN through the hybrid energy storage systems which is a combination of a battery and a capacitor. The supplied energy is consumed by the nodes to perform routing and using CEAR protocol the best path which reduces the power consumption and the cost is selected and the data packets get transmitted through 
such paths efficiently.

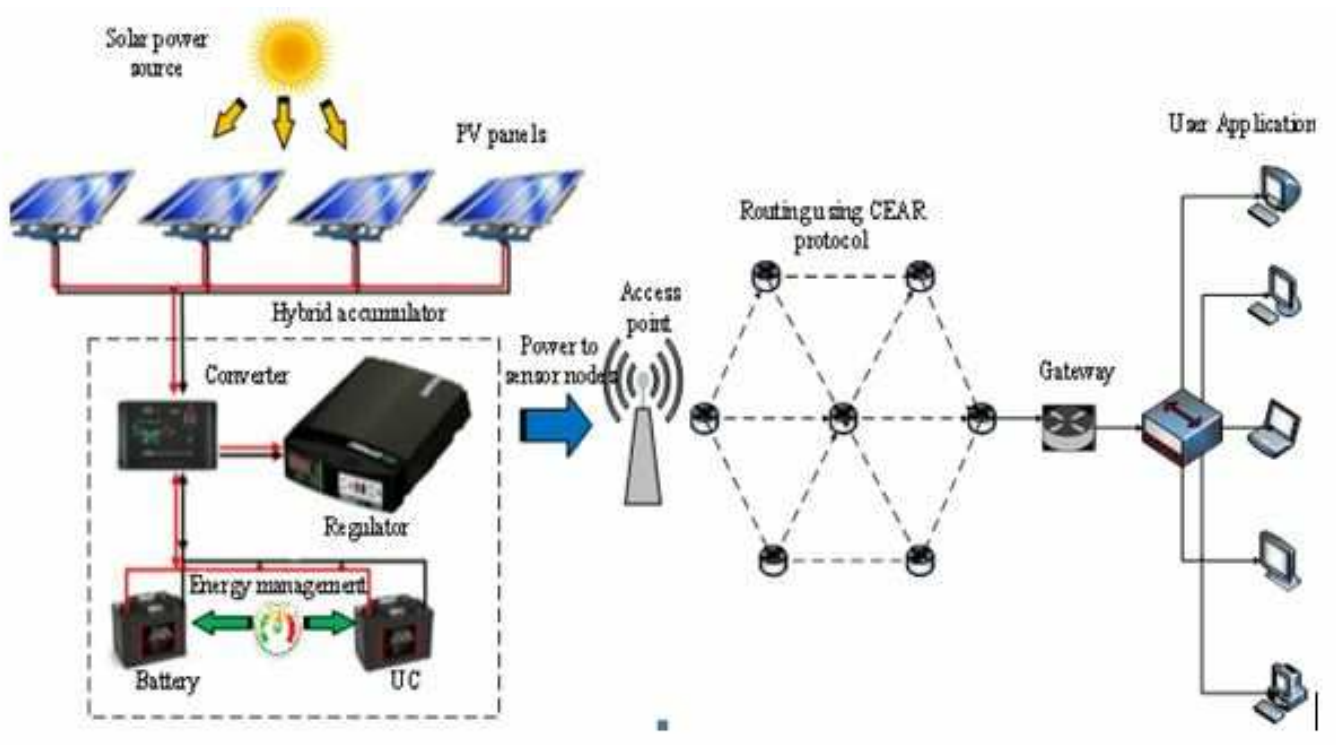

Figure 1: Architecture of our proposed method

\subsection{Powering up of WSN nodes by HESS model}

The HESS energy model considers the battery cycle life as well as a hybrid energy harvesting system. Also we propose a cost welfare and route source metric that takes into account the nodes' battery residual energy, harvesting rate and energy requirement to route the packet. A battery and an ultra-capacitor (UC) which is combined in the hybrid energy storage system do not have a regular connection always and also there is no direct connection between the UC and the harvesting unit. When a battery exceeds its life cycle then the node will be considered as dead. The battery is recharged when its residual energy falls below a pre-set threshold (1-D) $\mathrm{u}_{B}$ where $\mathrm{D}$ is "Depth of Discharge (DoD)" and $\mathrm{u}_{B}$ is the maximum capacity of the battery. Let $\zeta_{k}$ denote the arrival time of the $\mathrm{k}$-th packet request at a node. If the routing protocol selects the node, the node relays the packet. The period of activity consists of route selection and relaying of packet, in which the node does not harvest energy is assumed to be $\zeta_{p}$ seconds long. We define $\zeta_{k}{ }^{+}=\zeta_{k}+$ $\zeta$ p. The node harvests energy during the remainder of the time slot, which $\zeta_{h}$ seconds long. It follows that $\zeta_{k+1}=\zeta_{k}{ }^{+}+\zeta_{h}$. The residual energy of battery and UC for our models is described as follows.

$$
\begin{gathered}
U C_{A B L E}=E_{U C}^{\wedge}\left(n, \zeta_{k}\right)-l(j) E(n, R(j))>0 \\
B_{A B L E}=E_{B}^{\wedge}\left(n, \zeta_{k}\right)-(1-D) u_{B}-l(j) E(n, R(j))>0 \\
B_{R E C H A R G E}=E_{B}^{\wedge}\left(n, \zeta_{k}\right) \leq(1-D) u_{B}
\end{gathered}
$$

Where $U C_{A B L E}$ is the time in which the UC has enough energy to route the packet. $B_{A B L E}$ is the time that the battery has enough energy to route the packet. $B_{R E C H A R G E}$ is the event that the battery has exceeded its depth of discharge and cannot accept further route requests until it has recharged. $E_{U C}^{\wedge}\left(n, \zeta_{k}\right)$ denotes the residual energy on the $\mathrm{n}^{\text {th }}$ node $\mathrm{UC}$ at time $\xi_{k}, \mathrm{l}(\mathrm{j})$ is the route for the $\mathrm{j}^{\text {th }}$ packet, $\mathrm{E}($.$) is the energy per bit required to fulfill the route \mathrm{R}(\mathrm{j}), E_{B}^{\wedge}\left(n, \zeta_{k}\right)$ denotes the residual energy on the $\mathrm{n}^{\text {th }}$ node battery at time $\xi_{k}$, and $U_{B}$ denotes the maximum capacity in joules of the battery. To define the switch states $\mathrm{S}_{1}$ and $\mathrm{S}_{2}$ for the $\mathrm{n}^{\text {th }}$ node at time 
$\xi_{k}$, we shall use the indicator function $\mathrm{I}(\mathrm{A})$ is 1 , if the condition $\mathrm{A}$ is true and $\mathrm{I}(\mathrm{A})$ is 0 , when $\mathrm{A}$ is false. We observe that $S_{1}=1$ only, if the node is selected to relay the packet. Which implies

$$
S_{1}\left(n, \zeta_{k}\right)=I\left\{R E L A Y \cap\left(U C_{A B L E} \operatorname{cup} B_{A B L E}\right\}\right.
$$

$\mathrm{S}_{2}=0$ under different conditions depending on if the node is harvesting or transmitting.

$$
S_{2}\left(n ., \zeta_{k}\right)=1-I\left(\overline{U C_{A B L E}} \cap B_{A B L E} \cap R E L A Y\right)-I\left(B_{A B L E} \cap \overline{R E L A Y)}\right.
$$

The first indicator function can be 1 , if the node is chosen to relay in which case the second indicator function must be zero. The updated equations for the $\mathrm{UC}$ at time $\xi_{k-1}$ to time $\xi_{k}$ because of leaking, harvesting and loading is given below.

$$
\begin{gathered}
E_{U C}^{\wedge}\left(n, \zeta_{k}\right)=\min \left[\left(1-\alpha\left(\zeta_{k-1}, \zeta_{k}\right)\right) E_{U C}\left(n, \zeta_{k-1}^{+}\right)+S_{2}\left(n, \zeta_{k-1}^{+}\right) \gamma_{n}\left(\zeta_{k-1}^{+}, \zeta_{k}\right), u_{U C}\right. \\
E_{\text {load,UC}}^{\wedge}\left(n, \zeta_{k}, R(j)\right)=l(j) E\left(n, R(j) S_{1}\left(n, \zeta_{k}\right) S_{2}\left(n, \zeta_{k}\right)\right. \\
E_{U C}\left(n, \zeta_{k}^{+}\right)=\beta\left(n, D, \zeta_{k}\right)\left[E_{U C}^{\wedge}\left(n, \zeta_{k}\right)-E_{l o a d, U C}^{\wedge}\left(n, \zeta_{k}, R(j)\right)\right]
\end{gathered}
$$

Where $\alpha\left(\zeta_{k-1}, \zeta_{k}\right)$ denotes the time invariant fraction of energy leaked in the UC over a time slot, $E_{\text {load }, U C}^{\wedge}\left(n, \zeta_{k}, R(j)\right)$ is the energy consumed by a packet if the UC is used, $u_{U C}$ denotes the maximum capacity in joules of the $\mathrm{UC}$ and $\gamma_{n}\left(\zeta_{k-1}^{+}, \zeta_{k}\right)$ denotes the energy harvested at the $\mathrm{n}$-th node during time slot k-1. $E_{U C}\left(n, \zeta_{k}^{+}\right)$denotes the residual energy on the $\mathrm{n}^{\text {th }}$ node $\mathrm{UC}$ at $\zeta_{k}^{+}$and $\beta\left(n, D, \zeta_{k}\right)$ is an indicator function for the event that the battery on node $\mathrm{n}$ has not exceeded its finite cycle life at the beginning of time slot $\mathrm{k} . \beta\left(n, D, \zeta_{k}\right)$ is a non-increasing function of $s \xi_{k}$ and for a fixed $\xi_{k}$, is a strictly decreasing function of $\mathrm{D}$, the depth of discharge on the battery. Similarly the update equations of battery from time $\xi_{k-1}$ to time $\xi_{k}$ because of leaking, harvesting and loading is given below.

$$
\begin{gathered}
E_{B}^{\wedge}\left(n, \zeta_{k}\right)=\min \left\{\left[(1-\varphi) E_{B}\left(n, \zeta_{k-1}^{+}\right)\right]+\left[1-S_{2}\left(n, \zeta_{k-1}^{+}\right)\right] \gamma_{n}\left(\zeta_{k-1}^{+}, \zeta_{k}\right) u_{B}\right\} \\
E_{\text {load,B }}^{\wedge}\left(n, \zeta_{k}, R(j)=l(j) E(n, R(j)) S_{1}\left(n, \zeta_{k}\right) \cdot\left[1-S_{2}\left(n, \zeta_{k}\right)\right]\right. \\
E_{B}\left(n, \zeta_{k}^{+}\right)=\beta\left(n, D, \zeta_{k}\right)\left[E_{B}^{\wedge}\left(n, \zeta_{k}\right)-E^{\wedge}{ }_{\text {load }, B}\left(n, \zeta_{k}, R(j)\right)\right]
\end{gathered}
$$

Where $\varphi$ denotes the time varying fraction of energy leaked in the battery in one time slot $E^{\wedge}{ }_{\text {load,B }}\left(n, \zeta_{k}, R(j)\right)$ is the energy consumed by a packet if the battery is used and $E_{B}\left(n, \zeta_{k}^{+}\right)$ denotes the residual energy on the $\mathrm{n}^{\text {th }}$ node battery at $\zeta_{k}^{+}$.

\subsection{Routing in WSN}

Routing of packets with low power consumption and low cost is a major contribution in the wireless sensor networks. In order to perform such routing, a routing protocol is needed to be designed which is cost and energy aware. In our proposed method, the major challenges in the WSNs can be implemented by the CEAR protocol. It presents an efficient and reliable routing protocol those routes the packets to the sink node. In our proposed method, the sensor nodes present in the WSN utilizes the energy supplied by the hybrid system that carries the power from the renewable source.

\section{Setup phase}

The setup phase of the CEAR protocol handles the routing table replenishment of every node. It is loaded with no less than a route to the sink node keeping in mind the end goal to route the sensing packets. This is continued with the broadcast of control packets that conveys route information concerning the sink node. 


\section{CEAR protocol}

In routing, the data packets are forwarded to the hubs in the network which is a special node with an additional communication technology used to route the packets to the Base station (BS). In all sensor network applications, reliable and fast delivery of messages with reduced cost should be a major requirements. The routing performed in the WSN using our proposed CEAR routing protocol is shown in figure 2 .

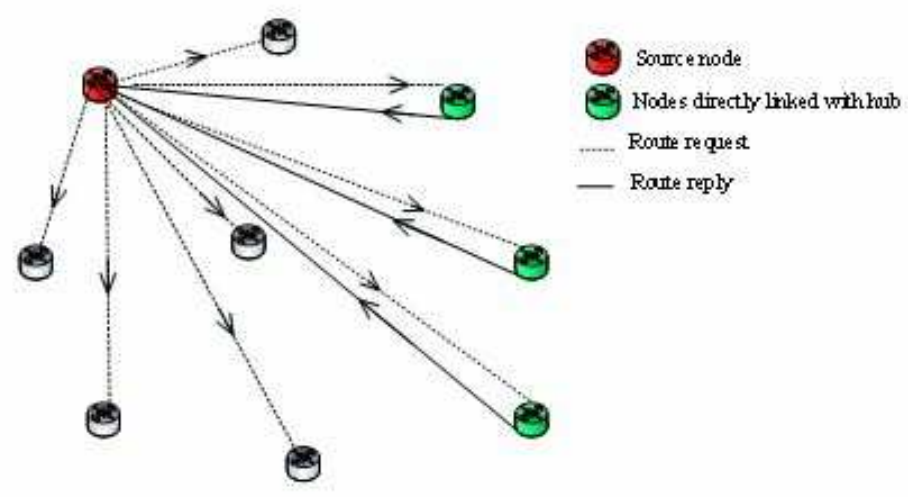

Figure 2: Routing in WSN using CEAR protocol

During data transmission, the source node transmits a Route Request (RREQ) packet to any destination hub. After the reception of packet, the destination hub transmits a Route Reply (RREP) packet to source hub. This process finds the nodes (selected nodes) that have linked with the hub which reduces the unwanted routing delay in the network which also delays the setup phase and the energy consumption. By means of this selected nodes the best path for routing is selected based on a Cost Function Request (CFR) and Cost Function Packet (CFP) which is shown in figure 3 .

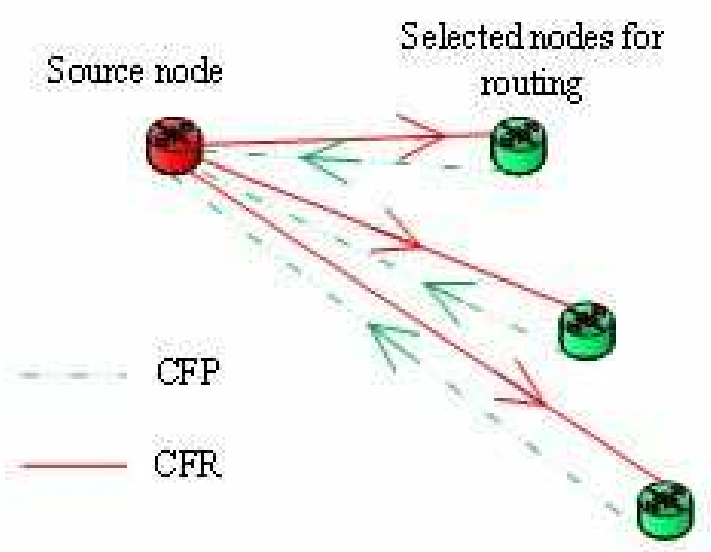

Figure 3: Path selection for routing based on CFP

To find an energy and cost efficient route from the selected nodes the source node again sends a Cost function request (CFR) to all the selected nodes. When the nodes receive the CFR, each node send a Cost function packet (CFP) which consist of the details of the cost welfare metric and the route source metric calculated for each node. 


\subsubsection{Cost welfare metric (CWM)}

Cost welfare metric helps the source node to select the node with lowest cost. To ensure, an extended lifetime for the network, the cost function must reflects the node ability to forward the packet through nodes with high UC residual energy and high harvesting energy rates. Cost welfare metric based on some parameters used for routing with low cost such as hop count, transmission queue, residual energy, harvested energy, life cycle of the battery which is given by the following equation

$$
\begin{array}{r}
C W M(n, \zeta)=\left(h c(n, \zeta) * w_{h c}+q_{o c}(n, \zeta) * w_{q o c}\right)-E_{U C}(n, \zeta) * w_{U C} \\
+E_{B}(n, \zeta) * w_{B}+\gamma(n, \zeta) * w_{\gamma}+L_{C}(n, \zeta) * W_{L C}
\end{array}
$$

Where hc is the hopcount which represents the minimum path length between the source node and the hub/sink node. $\mathrm{E}_{U C}$ and $\mathrm{E}_{B}$ represent the $\mathrm{UC}$ and battery residual energies in Joules. $\gamma$ is the harvested energy the node scavenged in the last time slot. $L c$ represents the battery cycle lifetime. $q_{o c}$ is the length for the node's transmission queue occupation. The hop count and transmission queue parameters are called undesirable parameters and the remaining parameters residual energy, harvested energy and battery cycle life are called as benefit parameters which supports the extension of network. The value of each parameters vary from 0 to 100 . The weight function for each parameter is denoted as $\mathrm{w}_{h c}, \mathrm{w}_{q o c}, \mathrm{w}_{U C}, \mathrm{w}_{B}, \mathrm{w}_{\gamma}, \mathrm{w}_{L c}$ this allows the priority scheme in the cost metric calculation. The weight limits for each parameter is set as follows,

$$
\begin{gathered}
W_{h c}+W_{q o c}=1 \\
W_{U C}+W_{B}+W \gamma+W_{L c}=1
\end{gathered}
$$

Where $\mathrm{w}_{h c}$, is the weight distribution of the hopcount, $\mathrm{w}_{\text {qoc }}$ is the weight distribution of the transmission queue, $\mathrm{W}_{U C}$ is the weight distribution of the ultra-capacitor, $\mathrm{w}_{B}$ is the weight distribution of the battery, $\mathrm{w}_{\gamma}$ is the weight distribution of the harvested energy, $\mathrm{w}_{L c}$ is the weight distribution of the lifecycle of battery. The weight distribution for such parameters based upon the above equations (13), (14) is given in the below table 1.

Table 1: Weight distribution for the parameters used in cost function metric

\begin{tabular}{|l|l|l|}
\hline Characteristics & Parameters & Weight distribution \\
\hline Positive & UC & 0.45 \\
& Battery & 0.2 \\
& Harvested energy & 0.15 \\
& Life cycle & 0.2 \\
\hline Negative & Hop count & 0.5 \\
& Transmission queue & 0.5 \\
\hline
\end{tabular}

The battery characteristics (residual energy and cycle life) are assigned with an importance of $20 \%$, while the harvesting energy has weight of $15 \%$. The two "negative" (cost) parameters share responsibility of $50 \%$ of in the cost function result. WSN applications have lower harvesting energy rates that can assign higher weight to $\mathrm{w} \gamma$, in order to customize the data flow. This formulation increases the flexibility of the cost function metric.

\subsubsection{Route score metric}

Route score metric based on energy level and weight assigned to the next hope node, link quality and weight assigned to the next hope node which is given by

$$
\text { Routescore }=P_{E} \cdot W_{E}+P_{L} \cdot W_{L}
$$


Where PE is the energy level of the next hope node, WE is the assigned weight for PE, PL is the link quality to the next hope node, and WL is the assigned weight for PL. The Route Score takes values from 0 to 100 . Higher values indicate a better route and the packets are usually routed through nodes with higher route Score metric value. The nodes with higher route score is selected as best nodes for routing and the packets are transmitted through such nodes. The route score value of each node will be stored in the routing table and that must be refreshed at each time slot in order to choose the best node. The refreshment of route score will be done by sending a feedback packet from the neighbours with the packet length of one byte for each time slot.

\section{Low energy and low cost routing}

By the above two metric calculations CEAR routing protocol chooses the best route that consumes less power and cost. The HESS metric is zero for a node that has sufficient energy on its UC to route a packet. If the UC has insufficient energy, then a non-zero value will be calculated for HESS based on the energy state of the battery. The HESS metric depends on the cost capacity metric of, which treats battery with interminable cycle life and $100 \%$ profundity of release. Be that as it may, we have to likewise have a segment of cost connected with spending the battery cycle life; for this, we can see the cycle life of a battery correspondingly to the life of a non-rechargeable battery. Consequently, we characterize two energy depletion functions for the battery one to demoralize choice of a node that is close to its predefined profundity of release, and another to discourage utilization of a node that is close to the end of its battery cycle life. The general cost capacity ought to increment, if both of these cost segments are increased. Let us first consider the cost component for discharge within one cycle, calculate energy depletion exponent be defined as follows

$$
\lambda_{B}\left(n, \zeta_{k}\right)=\frac{U_{B}-E_{B}^{\wedge}\left(n, \zeta_{k}\right)}{D_{u_{B}}}
$$

In words, the above calculated value will be zero, when the battery is fully charged and is one, when it is discharged down to its specified level of discharge. Next, the "cost component" for the within-cycle discharge is defined as follows

$$
C_{H E S S}\left(n, \zeta_{k}, R(j)\right)=\frac{D_{u_{B}}}{\left(\gamma_{n}+\varepsilon\right) \log \mu} \cdot\left(\mu^{\lambda_{B}\left(n, \zeta_{k}^{+}\right)}-1\right) l(j) \cdot E(n, R(j))
$$

\section{Results and discussions}

This section provides the simulation results and the comparison of our proposed method with the existing methods by evaluating various performance measures. The proposed method is implemented in MATLAB/Simulink platform. The proposed System configuration is given below:

Operating System: Windows 8; Processor: Intel Core i3; RAM: 4 GB; Platform: MATLAB/Simulink.

\subsection{Simulation results}

The simulation results obtained by our proposed method are presented in this section. Our proposed work consists of two phases which is carried out in two platforms. The simulation parameters utilized by the PV panels are given in table2 and the Specification of the other parameters used in storage system and WSN are given in table 3 . 
Table 2: Parameters of the PV panel

\begin{tabular}{|l|l|}
\hline Parameters & Specification \\
\hline Maximum power(W) & 100 \\
\hline Maximum current(A) & 5.86 \\
\hline Short circuit current(A) & 6.44 \\
\hline Open circuit current(A) & 21.4 \\
\hline Number of cells & 36 \\
\hline Weight $(\mathrm{kg})$ & 12 \\
\hline Length $(\mathrm{mm})$ & 1490 \\
\hline Depth $(\mathrm{mm})$ & 35 \\
\hline
\end{tabular}

Table 3: Specifications of the simulation parameters

\begin{tabular}{|l|l|l|l|}
\hline Storage system Parameters & Specification & WSN Parameters & Specification \\
\hline Converter efficiency & $80 \%$ & Protocol & CEAR \\
\hline UC leakage current & $1 \mathrm{~mA}$ & Sampling time & $5 \mathrm{us}$ \\
\hline Capacity of the battery & $20 \%$ & Traffic type & CBR \\
\hline Charge current & $300 \mathrm{~mA}$ & Simulation time & $600 \mathrm{sec}$ \\
\hline Discharge current & $150 \mathrm{~mA}$ & Number of nodes & $30,50,75,100$ \\
\hline Reference DC link voltage & $300 \mathrm{~V}$ & Simulation area & $500^{*} 500 \mathrm{~m}$ \\
\hline Nominal battery voltage & $144 \mathrm{~V}$ & Packet size & $512 \mathrm{MB}$ \\
\hline Nominal UC voltage & $125 \mathrm{~V}$ & Transmission range & $250 \mathrm{~m}$ \\
\hline Switching frequency & $15 \mathrm{KHz}$ & Node speed & $20 \mathrm{~m} / \mathrm{s}$ \\
\hline
\end{tabular}

The initial process of our research work is the extraction of energy from the solar panels and harvesting the energy to provide supply to the WSN. Our objective of proposed method is to extract the maximum amount of energy from the available solar panel and manage it by means of a storage device and then supply power to the sensor nodes. The energy obtained from the solar is based on the intensity of the light. As the intensity is high means greater voltage is obtained from the solar panel. The HEES stores the energy obtained from the solar panel and which is utilized for powering up the sensor nodes in the WSN.

The harvested solar energy is interfaced with our hybrid accumulator by the use of a converter placed between the solar panels and the accumulator. The current and voltage signals obtained by the converter are shown in figure 4(b) and (c). The energy from the harvesting unit reaches the storage unit which is the combination of both the battery and an ultra capacitor. The charging mode output of the storage unit is shown in figure 4(c). The terminal voltage obtained by the storage unit indicates both the combination of the capacitor and battery voltage. The terminal voltage obtained by the storage unit is shown in figure $4(\mathrm{~d})$.SOC is normally used when discussing the current state of a battery in use. An alternate form of the same measure is the depth of discharge (DoD), the inverse of SOC. The state of charge of the battery used in our proposed architecture is shown in figure 4(e).Then the energy output from the solar panel is stored efficiently by HEES which can be further utilized for wireless sensor powering process.

The resulting power from HEES is utilized by the wireless sensor network to perform a cost and energy aware routing. The simulation is carried out in 4 different scenarios by varying the number of nodes connected in the network. The results obtained by the proposed routing protocol with different number of nodes is given the below in table 4. The simulation is carried out in 4 different scenarios by varying the number of nodes connected in the network such as 30, 50, 75 and 100 .The energy harvested from the solar panel by utilizing hybrid accumulator is utilized to power up the sensor nodes in the WSN.Inturn WSN utilizes the energy for routing 


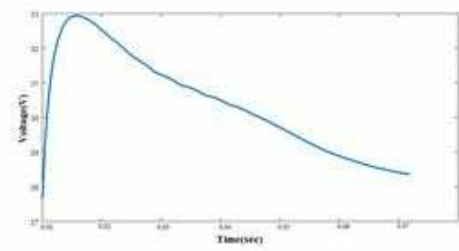

(a)

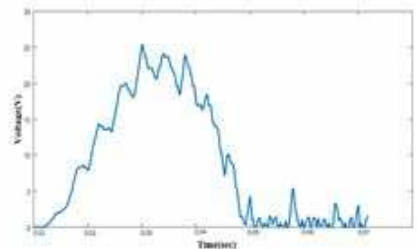

(b)

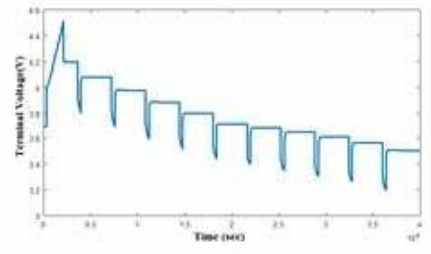

(e)

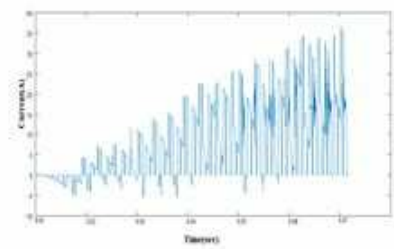

(b)

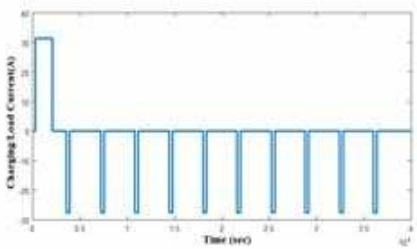

(d)

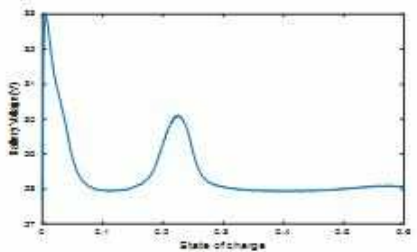

(f)

Figure 4: Energy obtained from Solar Panel,(a) Voltage obtained by the solar panel, (b) Current obtained by Boost converter, (c) Voltage obtained by boost converter (d) Charging of storage unit, (e) Terminal voltage of the storage unit, (f)State of charge of the battery

process by selecting best node from other sensor nodes for routing which is done by utilizing a cost and energy aware routing protocol. The simulation results of selecting the best node by the source node among all other sensor nodes by our proposed methodology is given below,

Thus the WSN source nodes which get supplied with the energy harvested from the solar panel is utilized for routing process which is done by utilizing the cost and energy aware routing protocol. Initially, the source node analysis with the 30 nodes based on the CEAR protocol and alternatively it searches to best nodes through the protocol along 50.75 and 100 nodes and finally chooses the best one among them and continuous the routing process. The energy is efficiently utilized by means of CEAR protocol for selecting best node for routing.

Table 4: Simulation results of CEAR protocol

\begin{tabular}{|l|l|l|l|l|}
\hline Number of nodes & $\mathbf{3 0}$ & $\mathbf{5 0}$ & $\mathbf{7 5}$ & $\mathbf{1 0 0}$ \\
\hline Packet delivery ratio & 2.651 & 2.891 & 2.912 & 2.9867 \\
\hline Average E2E delay & 110.442 & 117.981 & 121.157 & 127.982 \\
\hline Packet Loss & 200 & 210 & 213 & 219 \\
\hline Packet Loss Ratio & 0.953 & 1.237 & 1.567 & 1.864 \\
\hline Average throughput & 210.225 & 212.872 & 218.902 & 221.086 \\
\hline Energy consumption & 97 & 95 & 90 & 85 \\
\hline
\end{tabular}

The various parameters obtained by the simulations performed in different scenario that is, with different number of nodes such as 30, 50, 75, and 100 is shown in Figure 9.

The simulation results shows that, our proposed routing protocol selects the best node from the total available nodes and forward the data with high efficiency and low cost. Above 


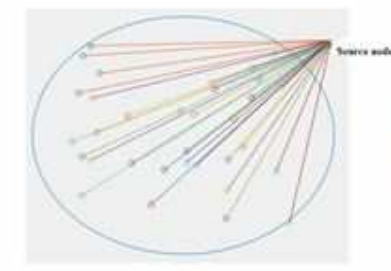

(a)

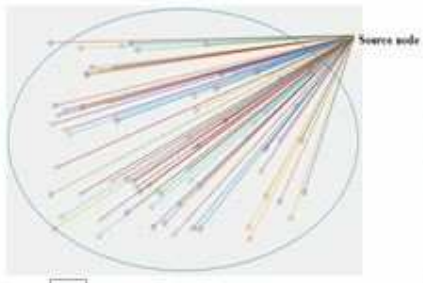

(c)

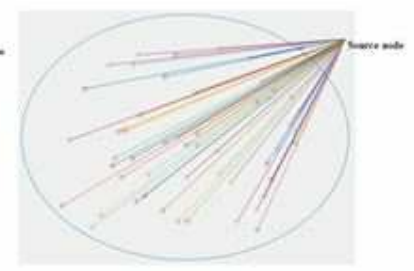

(b)

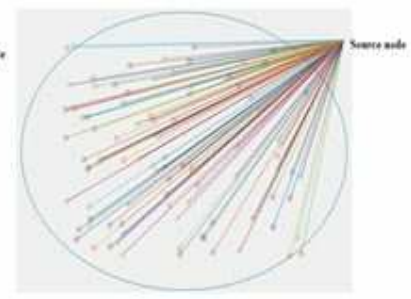

(d)

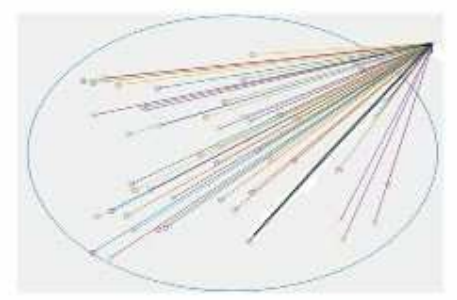

(e)

Figure 5: Simulation output for selecting best node by alternate path analysis (a) With 30 nodes, (b) With 50 nodes, (c) With 75 nodes, (d) With 100 nodes and (e) Selection of best node.

results are the evident to show the efficiency of our proposed CEAR protocol. In order to justify our proposed protocol is better than the existing protocols. The performance of the different protocols are calculated in terms of different parameters and compared in the upcoming sections.

\subsection{Performance Evaluation of CEAR:}

The performance of the protocol depends on various parameters and chosen for simulation. The main parameters are packet size, no of nodes, transmission range and the structure of the network. Based on the above parameters the following evaluation is calculated.

\section{Packet delivery ratio (PDR):}

The packet delivery ration is defined as the performance measure of routing protocol in any network in terms of packet delivery level. If PDR is high, then the performance is better. The relation for packet delivery ratio is as follows

$$
\text { Packet delivery ratio }=\frac{\sum(\text { Total packets received by all destination node })}{\sum(\text { Total packets send by all source node })}
$$

\section{Average End-to-End Delay:}

The average end-to-end delay can be obtained by computing the mean of end-to-end delay of all successfully delivered messages. If the distance between source and destination increases, 

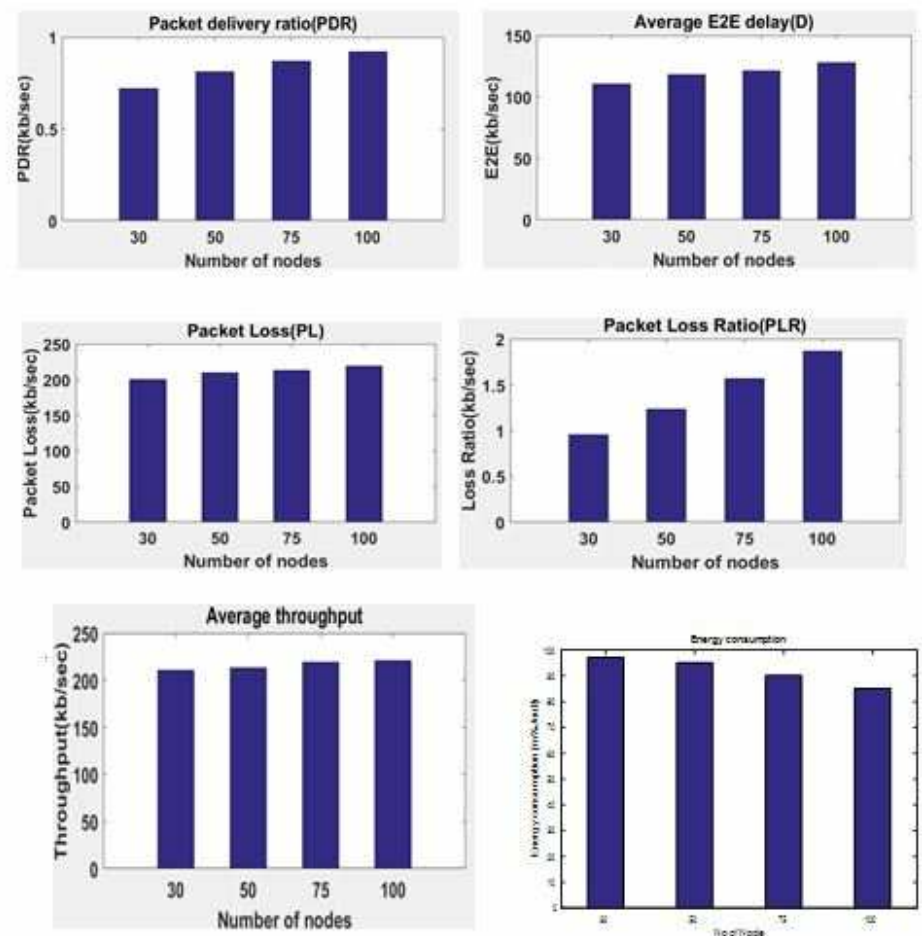

Figure 6: Simulation Results of CEAR protocol in terms of PDR, E2E delay, Packet loss, PLR and Throughput and the energy consumption.

then the probability of packet drop is also increases. The Average End-to-End Delay is expressed as follows

$$
D=\frac{1}{n} \sum_{i=1}^{n}\left(T_{r_{i}}-T_{S_{i}}\right) * 1000[m s]
$$

Where $\mathrm{D}$ is the average end to end delay, I is the packet identifier, $\mathrm{n}$ is the number of packets successfully delivered, $T_{r_{i}}$ is the reception time and $T_{S_{i}}$ is the send time.

\section{Packet Loss:}

Packet Loss is the ratio of the number of packets that never reached the destination to the number of packets originated by the source which are given by

$$
P L=\frac{(\text { SSentPackets }- \text { nReceivedPackets })}{n \text { SentPackets }}
$$

\section{Packet Loss Ratio:}

Packet Loss Ratio is the percentage of ratio of the number of packets that never reached the destination to the number of packets originated by the source (PL*100) which is given by

$$
P L R=\frac{(n \text { SentPackets }- \text { nReceivedPackets })}{n \text { SentPackets }} * 100
$$




\section{Average Throughput:}

It is the average of the total throughput. It is also measured in packets per unit Time Interval Length (TIL).

$$
\text { Average throughput }=\frac{\text { Received size }}{(\text { Stoptime }- \text { Start time })} *\left(\frac{8}{1000}\right)
$$

\subsection{Comparison Results}

The use of HESS in our proposed routing method is suggested due to the better storage capacity, efficiency, energy density, power density, response time, cycle life time and cost when compared to other storage devices used in the existing methods. The importance of our proposed HESS in our proposed routing protocol is shown by the below table 3 .

Table 5: Comparison of several Energy Storage Systems

\begin{tabular}{|l|l|l|l|l|l|l|}
\hline & & & & & $\begin{array}{l}\text { Hybrid } \\
\text { ESS (proposed) }\end{array}$ \\
\hline Efficiency (\%) & $60-80$ & $95-98$ & 95 & 95 & 70 & $98-99.9$ \\
\hline Energy density (Wh/Kg) & $20-200$ & $30-100$ & $5-50$ & 50 & 120 & $30-200$ \\
\hline Power density (W/Kg) & $25-1000$ & $10-1000$ & 2000 & 4000 & 120 & 4500 \\
\hline Response time (ms) & 30 & 5 & 5 & 5 & 100 & 3 \\
\hline Cycle life (time) & $200-2000$ & 1000 & 20000 & 50000 & 2000 & 50000 \\
\hline Cost (S/kW h) & $150-1300$ & 2000 & $380-2500$ & $250-350$ & 450 & $100-150$ \\
\hline
\end{tabular}

The comparison graph between HESS and other existing energy storage devices regarding the above table is shown in figure 7 .

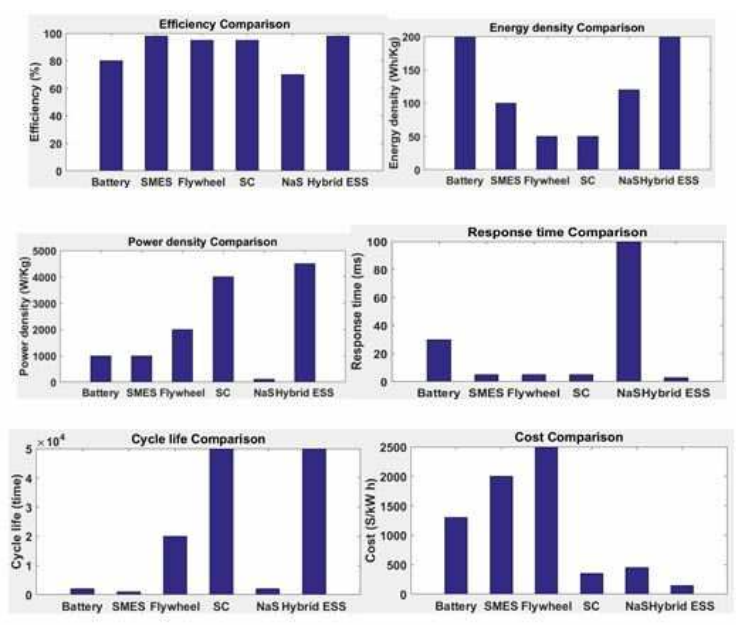

Figure 7: Comparison Results for Efficiency, Energy density, Power density, Response time, Cycle life time, Cost

The figure 7 shows the comparison graph, the efficiency of the HESS is likely to be the same when comparing to the SMES, Flywheel, and Super capacitor. But when comparing in all other factors such as energy density, power density, cycle life, response time, cost HESS is proved to be better solution. We can conclude that the use of HESS in our proposed routing provides better solution and improves the overall performance of the network. By the utilization of the HESS in our proposed routing protocol selects the path with best nodes that consumes less power and low 
cost. Ad hoc On-Demand Distance Vector (AODV), Proactive Source Routing (PSR), Greedy Perimeter Stateless Routing (GPSR) are compared with our proposed routing protocols is shown below in table 6 .

Table 6: Comparison of our proposed routing protocol with existing protocols

\begin{tabular}{|l|l|l|l|l|l|l|l|}
\hline $\begin{array}{l}\text { Number } \\
\text { of } \\
\text { Nodes }\end{array}$ & $\begin{array}{l}\text { Type of } \\
\text { proting } \\
\text { protols }\end{array}$ & $\begin{array}{l}\text { Packet } \\
\text { Loss }\end{array}$ & $\begin{array}{l}\text { Average } \\
\text { E2E } \\
\text { Delay }\end{array}$ & $\begin{array}{l}\text { Packet } \\
\text { Delivery } \\
\text { Ratio }\end{array}$ & $\begin{array}{l}\text { Average } \\
\text { Throughput }\end{array}$ & $\begin{array}{l}\text { Packet } \\
\text { Loss } \\
\text { Ratio }\end{array}$ & $\begin{array}{l}\text { Energy } \\
\text { consump- } \\
\text { tion }\end{array}$ \\
\hline 30 & AODV & 248 & 120.442 & 9.3671 & 207.864 & 2.890 & 99 \\
& PSR & 246 & 127.754 & 70.809 & 210.56 & 1.590 & 99.2 \\
& GPSR & 235 & 110.750 & 70.809 & 218.56 & 1.050 & 99.56 \\
& Proposed & 205 & 109.543 & 93.608 & 240.9 & 1.152 & 97 \\
\hline 50 & AODV & 644 & 131.145 & 2.891 & 212.872 & 2.890 & 96 \\
& PSR & 173 & 74.7002 & 40.4567 & 214.55 & 1.678 & 97.25 \\
& GPSR & 160 & 70.7008 & 45.1786 & 248.55 & 1.150 & 98.32 \\
& Proposed & 210 & 117.981 & 93.6028 & 240.9 & 1.237 & 95 \\
\hline 75 & AODV & 799 & 130.306 & 1.4598 & 136.936 & 5.999 & 93 \\
& PSR & 383 & 193.11 & 10.99 & 150.9 & 1.909 & 94.25 \\
& GPSR & 280 & 110.90 & 11.4177 & 190.18 & 1.190 & 95.12 \\
& Proposed & 265 & 102.375 & 98.1747 & 240.58 & 1.143 & 90 \\
\hline 100 & AODV & 1285 & 129.825 & 1.2843 & 146.431 & 6.789 & 88 \\
& PSR & 313 & 142.524 & 10.99 & 150.9 & 1.909 & 89.21 \\
& GPSR & 280 & 110.90 & 1.2919 & 198.33 & 1.190 & 90.25 \\
& Proposed & 274 & 108.532 & 92.3664 & 266.87 & 1.678 & 89 \\
\hline
\end{tabular}

The figures 8 to 13 shows the comparison graphs in terms of the important parameters resulted by the routing protocol for different number of nodes regarding to the above comparison tables.

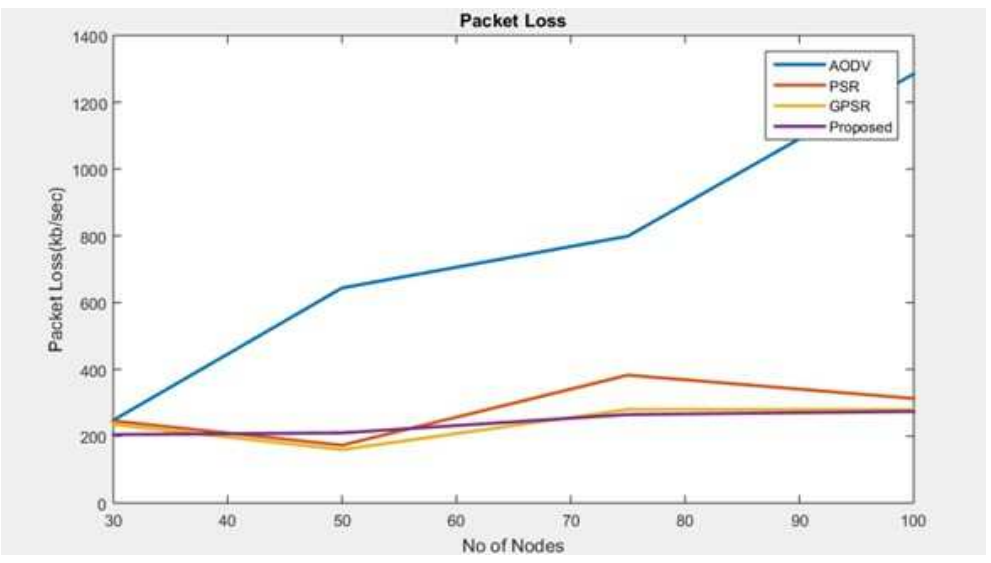

Figure 8: Packet Loss Comparison of proposed CEAR protocol with exisiting protocols

Figure 8 shows the packet loss comparison of different type of routing protocols with our proposed CEAR routing protocol with varying number of nodes. The packet loss denotes the inability of the path selected for transmission in the network. For a successive node the packet loss should be as minimum as possible. From the figure 11 it is visible that the packet loss of our proposed protocol is very less compared to other protocols. This makes us to say that our proposed protocol is best for routing in WSN.

Figure 9 shows the E2E delay comparison of different type of routing protocols with our proposed CEAR routing protocol with varying number of nodes. If the E2E delay exists in a 


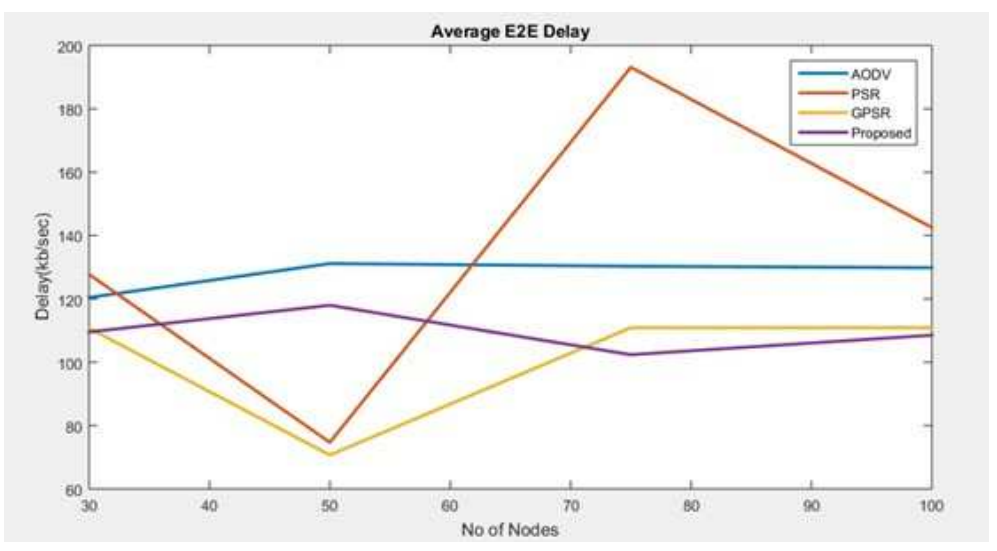

Figure 9: E2E delay Comparison of proposed CEAR protocol with exisiting protocols

network at a maximum range the performance of the overall network is degraded even though the power consumption and the cost of routing is low. From figure 12 we can demonstate that our proposed CEAR routing protocol produces minimal delay compared to other existing protocols.

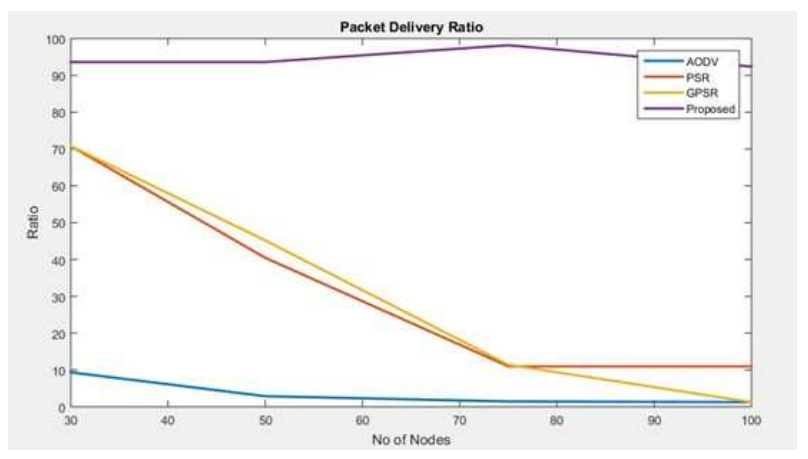

Figure 10: Packet delivery ratio Comparison of proposed CEAR protocol with exisiting protocols

Figure 10 shows the packet delivery ratio comparison of different type of routing protocols with our proposed CEAR routing protocol with varying number of nodes. Packet delivery ratio is the maximum ability of the network to deliver a packet from source to destination. From figure 13 it is evident that the packet delivery ratio of our proposed routing protocol is higher than the exisiting protocols which makes us to come a decision that we can use our proposed routing protocol to deliver maximum packets without loss in the network.

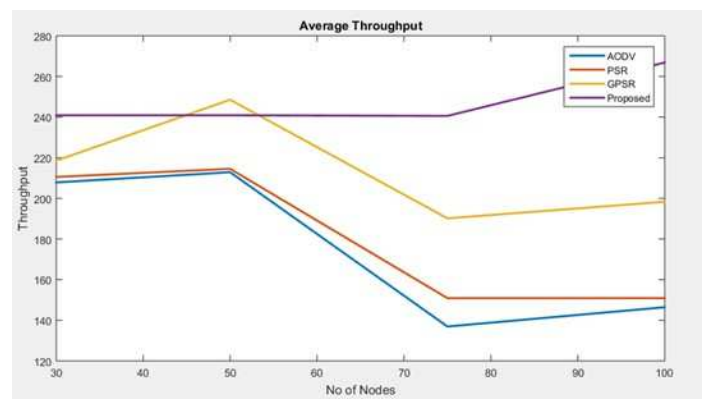

Figure 11: Throughput Comparison of proposed CEAR protocol with existing protocols

Figure 11 shows the throughput comparison of different type of routing protocols with our 


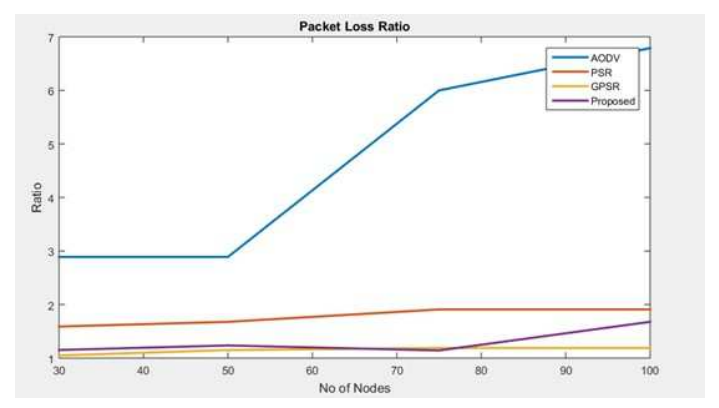

Figure 12: Packet loss Ratio Comparison of proposed CEAR protocol with exisiting protocols

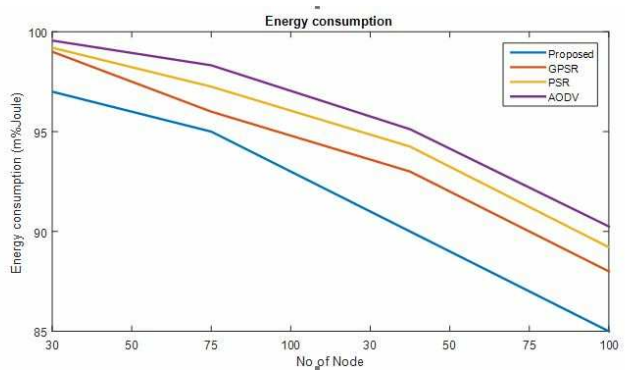

Figure 13: Energy consumption Comparison of proposed CEAR protocol with existing protocols

proposed CEAR routing protocol with varying number of nodes. For an efficient network routing protocol the throughput should be maximum as possible. From figure 11, it is shown that the proposed routing protocol results in high throughput than the existing protocols. From this we can come to know that our proposed routing protocol is efficient than the other existing protocols.

Figure 12 shows the packet loss ratio comparison of different type of routing protocols with our proposed CEAR routing protocol with varying number of nodes. Packet loss ratio indexes the variation between the number of packets send from the source to the number of packets received at the destination. The reduced packet loss ratio indicates the value of the routing protocol. It is clear that our proposed routing protocol produces less packet loss ratio which indicates that the number of delivered packets is maximum.From the figure 13 it is visible that the energy consumed by our proposed methodology is very less compared to that of other routing protocols. From the above results and comparison, it clearly shows that our proposed routing protocol selects the best node with less power consumption and low cost and also it shows that the utilization of HESS for routing by the sensor nodes in our proposed method is the most optimal solution to reduce the power consumption.

\section{Conclusion}

In this paper we have proposed energy and cost aware routing protocol used in wireless sensor network. The sensor nodes present in the WSN utilizes the power, which is generated by the renewable energy source such as solar energy by means of a storage system. The storage and management of the extracted energy is done by means of a hybrid storage device such as the combination of a battery and an ultra-capacitor (UC). The UC suits the primary buffer of the storage system by preventing the quick exhaustion of the battery cycle lifetime. By using a cost and energy aware routing protocol at the network level, we acquired a noticeable increment in the network residual energy without trading off the data packet delivery. Moreover in this 
paper we also analysis the performance of the network while transmission by means of number of parameters. From the results and comparison such as Packet loss, end to end delay, packet delivery ratio, throughput, packet loss ratio shows that the proposed method is a technically viable option among the available other existing techniques.

\section{Author contributions. Conflict of interest}

The authors contributed equally to this work. The authors declare no conflict of interest.

\section{Bibliography}

[1] Abeywardana, D.B.W.; Hredzak, B.; Vassilios, A.G. (2015). Single-Phase Grid-Connected LiFePO 4 Battery-Supercapacitor Hybrid Energy Storage System With Interleaved Boost Inverter, IEEE Transactions on Power Electronics, 30(10), 5591-5604, 2015.

[2] Bajpai, P.; Dash, V. (2012). Hybrid renewable energy systems for power generation in standalone applications: A review, Renewable and Sustainable Energy Reviews, 16(5), 2926-2939, 2012 .

[3] Cao, J.; Emadi, A. (2012). A new battery/ultracapacitor hybrid energy storage system for electric, hybrid, and plug-in hybrid electric vehicles, IEEE Transactions on Power Electronics, 27(1), 122-132, 2012.

[4] Choi, M.-E.; Kim, S.-W.; Seo, S.-W.(2012). Energy management optimization in a battery/supercapacitor hybrid energy storage system, IEEE Trans. Smart Grid, 3(1), 463-472, 2012.

[5] Das, D.; Roy, A.K.; Sinha, N. (2012). GA based frequency controller for solar thermal-dieselwind hybrid energy generation/energy storage system, International Journal of Electrical Power \& Energy Systems, 43(1), 262-279, 2012.

[6] Díaz-González, F.; Sumper, A.; Gomis-Bellmunt, O.; Villafáfila-Robles, R. (2012). A review of energy storage technologies for wind power applications, Renewable and Sustainable Energy Reviews, 16(4), 2154-2171, 2012.

[7] Erdinc, O.; Uzunoglu, M. (2013). Optimum design of hybrid renewable energy systems: Overview of different approaches, Renewable and Sustainable Energy Reviews, 16(3), 1412$1425,2013$.

[8] Etxeberria, A.; Vechiu, I.; Camblong, H.; Vinassa, J.M. (2012). Comparison of three topologies and controls of a hybrid energy storage system for microgrids, Energy Conversion and Management, 54(1), 113-121, 2012.

[9] Evans, A.; Strezov, V.; Evans, T.J. (2012). Assessment of utility energy storage options for increased renewable energy penetration, Renewable and Sustainable Energy Reviews, 16(6), 4141-4147, 2012.

[10] Glavin, M. E.; Hurley, W.G.(2012). Optimisation of a photovoltaic battery ultracapacitor hybrid energy storage system, Solar Energy, 86(10), 3009-3020, 2012.

[11] Guerrero, J.P.; Chiang, L.M.; Lee, T.-L.; Mukul Chandorkar, M. (2013). Advanced control architectures for intelligent microgrids - Part II: Power quality, energy storage, and AC/DC microgrids, IEEE Transactions on Industrial Electronics, 60(4), 1263-1270, 2013. 
[12] Huang, W.; Qahouq, J.A.A. (2015). Energy sharing control scheme for state-of-charge balancing of distributed battery energy storage system, IEEE Transactions on Industrial Electronics, 62(5), 2764-2776, 2015.

[13] Laldin, O.; Moshirvaziri, M.; Trescases, O. (2013). Predictive algorithm for optimizing power flow in hybrid ultracapacitor/battery storage systems for light electric vehicles, IEEE Transactions on power electronics, 28(8), 3882-3895, 2013.

[14] Lee, H.; Byoung, Y.S.; Han, S.; Jung, S.; Park, B.; Jang, G. (2012). Compensation for the power fluctuation of the large scale wind farm using hybrid energy storage applications, IEEE Transactions on Applied Superconductivity, 22(3), 5701904-570197, 2012.

[15] Li, X.; Dong, H.; Lai, X. (2013). Battery energy storage station (BESS)-based smoothing control of photovoltaic (PV) and wind power generation fluctuations, IEEE Transactions on Sustainable Energy, 4(2), 464-473, 2013.

[16] Makarov, Y.V.; Du, P.; Kintner-Meyer, M.C.W.; Jin, C.; Illian, H.F. (2012). Sizing energy storage to accommodate high penetration of variable energy resources, IEEE Transactions on sustainable Energy, 3(1), 34-40, 2012.

[17] Marano, V.; Rizzo, G.; Tiano, F.A. (2012). Application of dynamic programming to the optimal management of a hybrid power plant with wind turbines, photovoltaic panels and compressed air energy storage, Applied Energy, 97, 849-859, 2012.

[18] Mukherjee, N.; Strickland, D.; (2015). Control of second-life hybrid battery energy storage system based on modular boost-multilevel buck converter, IEEE Transactions on Industrial Electronics, 62(2), 1034-1046, 2015.

[19] Nanda, A; Rath, A.K. (2018). Fuzzy A-Star Based Cost Effective Routing (FACER) in WSNs, Progress in Advanced Computing and Intelligent Engineering, 557-563, 2018.

[20] Onar, O.C.; Khaligh, A. (2012). A novel integrated magnetic structure based DC/DC converter for hybrid battery/ultracapacitor energy storage systems, IEEE transactions on smart grid, 3(1), 296-307, 2012.

[21] Padyal, R.H; Kadam, S.V. (2017). Continuous neighbour discovery approach for improvement of routing performance in WSN, Convergence in Technology (I2CT), 2017 and International Conference for, 675-679, 2017.

[22] Gupta, S.K.; Kuila, P; Jana, P.K.(2017). GA Based Energy Efficient and Balanced Routing in k-Connected Wireless Sensor Networks, Proceedings of the First International Conference on Intelligent Computing and Communication, 679-686, 2017.

[23] Ren, G.; Ma, G.; Cong, N. (2015). Review of electrical energy storage system for vehicular applications, Renewable and Sustainable Energy Reviews, 41, 225-236, 2015.

[24] Selvi, M.; Velvizhy, P.; Ganapathy, S.; Nehemiah, H.K; Kannan, A.(2017). A rule based delay constrained energy efficient routing technique for wireless sensor networks, Cluster Computing, 1-10, 2017.

[25] Tan, X.; Li, Q.; Wang, H. (2013). Advances and trends of energy storage technology in microgrid, International Journal of Electrical Power \& Energy Systems, 44(1), 179-191, 2013. 
[26] Xie, Q.; Wang, Y.; Kim, Y.; Pedram, M.; Chang, N. (2013). Charge allocation in hybrid electrical energy storage systems, IEEE Transactions on Computer-Aided Design of Integrated Circuits and Systems, 32(7), 1003-1016, 2013.

[27] Zhang, B.; Simon, R.; Aydin, H. (2013). Harvesting-aware energy management for timecritical wireless sensor networks with joint voltage and modulation scaling, IEEE Transactions on Industrial Informatics, 9(1), 514-526, 2013.

[28] Zhao, H.; Wu, Q.; Hu, S.; Xu, H.; Rasmussen, C.N. (2015). Review of energy storage system for wind power integration support, Applied Energy, 137, 545-553, 2015.

[29] Zhou, Z.; Benbouzid, M.; Charpentier, J.F.; Scuille, F.; Tang, T. (2013). A review of energy storage technologies for marine current energy systems, Renewable and Sustainable Energy Reviews, 18, 390-400, 2013. 